hep-th/9811017

BROWN-HET-1150

\title{
Constraints on Higher Derivative Operators in Maximally Supersymmetric Gauge Theory
}

\author{
David A. Lowe \\ Department of Physics \\ Brown University \\ Providence, RI 02912, USA \\ lowe@het. brown.edu \\ and \\ Rikard von Unge \\ Department of Theoretical Physics and Astrophysics \\ Masaryk University \\ Kotlářská 2, 61137 Brno \\ Czech Republic \\ unge@physics.muni.cz
}

\begin{abstract}
Following the work of Dine and Seiberg for $S U(2)$, we study the leading irrelevant operators on the moduli space of $\mathcal{N}=4$ supersymmetric $S U(N)$ gauge theory. These operators are argued to be one-loop exact, and are explicitly computed.
\end{abstract}

October, 1998 


\section{Introduction}

The generic actions of field theories with supersymmetry are tightly constrained. In certain cases, supersymmetry completely determines the form of some of the terms in the action. For example with $\mathcal{N}=2$ in four dimensions supersymmetry fixes the entire action at leading order at low energies in terms of a $\mathcal{N}=2$ superspace chiral integral of a holomorphic function. For $\mathcal{N}=4$ we expect even stronger constraints, however analysis is hampered by the lack of a $\mathcal{N}=4$ superspace formalism. If such a formalism existed, a chiral integral would be an integral over eight Grassman coordinates and one would expect non-renormalization theorems for four derivative terms. It is nevertheless possible to work with $\mathcal{N}=2$ superfields and make additional symmetry arguments to constrain the form of the action.

This is the approach we take in this paper, where we prove a non-renormalization theorem for terms quartic in derivatives in theories with $\mathcal{N}=4$ supersymmetry, and terms related to these by supersymmetry. The terms of this type arise from an integral over all of $\mathcal{N}=2$ superspace of a real function of the superfields

$$
\int d^{8} \theta \mathcal{H}\left(W, \bar{W}, Y, \bar{Y}, \tau, \tau^{\dagger}\right)
$$

where $W$ is the field strength supermultiplet and $Y$ the adjoint hypermultiplet. The terms involving just the $\mathcal{N}=2$ vector multiplets have been much studied in previous works. In [1], it was shown that the perturbative contributions to $\mathcal{H}(W, \bar{W})$ must take the form

$$
\mathcal{H}(W, \bar{W})=\mathcal{H}^{0}+c\left(\log W^{2}+g^{0}(W)\right)\left(\log \bar{W}^{2}+g^{0}(\bar{W})\right)
$$

where $\mathcal{H}^{0}$ and $g^{0}$ are homogeneous and $c$ is a constant. For $S U(2) g^{0}=0$, and the one loop contribution to $\mathcal{H}^{0}$ was determined in [1]. The result can be understood as follows. $\mathcal{H}^{0}$ receives contributions only from the non-commuting off-diagonal degrees of freedom in the vector multiplet, the $\mathrm{W}$ bosons. These are all massive if we consider maximal symmetry breaking and so $\mathcal{H}^{0}$ is irrelevant if we are only interested in the massless degrees of freedom in the maximally broken case. The second term in (1.2) represents everything that does not go into $\mathcal{H}^{0}$. In particular, if all degrees of freedom are commuting, this term is all there is. This second term was considered further in [2], where it was argued that the one-loop contributions to $\mathcal{H}$ are exact non-perturbatively for $S U(2)$. 1 In this work, we generalize

1 In [3, 4] it was checked that this term does not receive non-perturbative contributions. The constant $c$ has recently been found to be $1 /(8 \pi)^{2}$ [5, 6, 7 . 
this result to the $S U(N)$ case and completely determine $\mathcal{H}$ for the case with maximally broken gauge symmetry.

This result is important in the context of recent studies in Matrix theory, and in the correspondence between string theory in an anti-de Sitter background and large $N S U(N)$ $\mathcal{N}=4$ supersymmetric Yang-Mills theory [8,9]. From the Matrix theory point of view, the non-renormalization of these terms leads to agreement with tree-level M-theory in discrete light-cone gauge further compactified on a three-torus. The result of [2], gives the needed non-renormalization theorem for the $S U(2)$ case and the complete non-renormalization theorem for general $S U(N)$ is obtained in the present work.

Discretized light-cone quantization of M-theory further compactified on $T^{3-n}$ is described by dimensionally reducing the four dimensional gauge theory to $4-n$ dimensions. In this case it is not obvious that the non-renormalization theorem will carry over because the underlying conformal invariance of the four dimensional theory is broken in lower dimensions. In three dimensions, instanton effects appear, with just the right structure predicted by the correspondence with supergravity for $S U(2)$ [10]. In one dimension, a non-renormalization theorem has been proven by very different methods for the supersymmetric $S U(2)$ quantum mechanics, where it is found that the one-loop contribution is exact [11]. For $S U(N)$ it has been established that the terms that appear at one-loop are not renormalized [12], however the possibility remains that other tensor structures can appear at the same order, beyond one-loop. As we will describe below, conformal invariance and chiral $U(1)_{R}$ symmetry is sufficient to rule out such extra contributions in four dimensions.

There is a qualitative difference between the $S U(N)$ and the $S U(2)$ case from the brane point of view. Consider one D-threebrane probing a generic background of $N-1$ others. For $N=2$ the velocity of the background D-brane may be set to zero, giving rise to a supersymmetric background. It is not surprising then that the metric induced by this brane is protected by supersymmetry. For $N>2$ on the other hand, the background will generically break all supersymmetry. Nevertheless the result we find is that the metric induced by this background is protected by supersymmetry.

\section{2. $F^{4}$ Terms in $\mathcal{N}=4$ SUSY $S U(N)$ Gauge Theory}

In the following, the $\mathcal{N}=2$ superspace formalism will be used. Each $\mathcal{N}=4$ vector multiplet consists of a $\mathcal{N}=2$ vector multiplet and a hypermultiplet in the adjoint representation. We will be interested in a generic point on the Coulomb branch of the theory where $S U(N)$ is broken to $U(1)^{N-1}$ modulo Weyl transformations. The light degrees of freedom therefore correspond to a collection of $N-1 \mathcal{N}=4$ multiplets. 
The $\mathcal{N}=4$ theory is finite and conformally invariant. For a suitable definition of the matrix of couplings $\tau_{i j}$ and field strength superfields $W_{i}$ compatible with duality, the generic gauge kinetic terms can be written as

$$
\operatorname{Im} \int d^{2} \theta d^{2} \tilde{\theta} \tau_{i j} W_{i} W_{j}
$$

where $\tau_{i j}=\tau C_{i j}$, where $\tau=\frac{\theta}{4 \pi}+\frac{2 \pi i}{g^{2}}$ is the coupling that transforms in the standard way under $S L(2, \mathbb{Z})$ duality and $C_{i j}$ is proportional to the Cartan matrix. In addition there are kinetic terms for the hypermultiplet superfields $Y_{i}$ and a superpotential coupling the vector and hypermultiplet which are completely determined by supersymmetry.

Now consider terms of the form (1.1). Scale invariance implies that $\mathcal{H}$ must be dimensionless, and since the theory is $U(1)_{R}$ invariant (there is no anomaly in $\mathcal{N}=4$ ) it must transform trivially under the chiral $U(1)_{R}$ symmetry. This implies strong constraints on the functions of the $W_{i}$ and $Y_{i}$ that can appear in $\mathcal{H}$. To determine the behavior as a function of $\tau$ we follow the argument of [13], and promote $\tau$ to a background vector superfield. Demanding scale invariance and $U(1)_{R}$ invariance in this situation implies that $\mathcal{H}$ cannot depend on $\tau$ at all. We find therefore $\mathcal{H}$ must be one-loop exact.

It remains then to explicitly determine the one-loop form of $\mathcal{H}$. For the moment we will assume that only the scalars in the $\mathcal{N}=2$ vector multiplets have nontrivial expectation values. The one-loop form for $\mathcal{H}(W, \bar{W})$ was determined for a general $\mathcal{N}=4$ super YangMills theory in terms of a momentum integral in [6]

$$
\mathcal{H}(W, \bar{W})=\frac{1}{2} \int \frac{d p^{2}}{(4 \pi)^{2} p^{2}} \operatorname{Tr}_{A} \log \left(1+\frac{W \bar{W}+\bar{W} W}{2 p^{2}}\right),
$$

where the trace is in the adjoint representation which we denote by the subscript $A$.

It is more convenient to use the fundamental representation to describe a generic point on the Coulomb branch. The generators of the fundamental representation (denoted by subscript $F$ ) are related to those of the adjoint representation by

$$
\left(T_{A}^{a}\right)_{j ; l}^{i k}=\left(T_{F}^{a}\right)_{l}^{i} \delta_{j}^{k}-\left(T_{F}^{a}\right)_{j}^{k} \delta_{l}^{i}
$$

Here $a$ runs over the group generators and $i, j, k, l$ are indices in the fundamental. The scalar part of the field $W$ has an expectation value that lives in the Cartan subalgebra

$$
W_{j ; l}^{i k}=\left\langle W^{a}\right\rangle\left(\left(T_{F}^{a}\right)_{i}-\left(T_{F}^{a}\right)_{k}\right) \delta_{l}^{i} \delta_{j}^{k}
$$

The $T_{F}$ 's appearing here are diagonal, so are just written with a single fundamental index. 
Inserting (2.4) into the expression that appears in the integral (2.2) leads to

$$
(W \bar{W}+\bar{W} W)_{j ; l}^{i k}=2 \delta_{l}^{i} \delta_{j}^{k}\left(W_{i}-W_{k}\right)\left(\bar{W}_{i}-\bar{W}_{k}\right)
$$

where $W_{i}=W^{a}\left(T_{F}^{a}\right)_{i}$ are diagonal. Now the point is that the right-hand side of (2.5) is diagonal in adjoint indices (the trace of (2.3) involves the contraction of $i$ with $l$ and $j$ with $k$ ). Evaluating the integral (2.2) gives

$$
\mathcal{H}(W, \bar{W})=\frac{1}{(4 \pi)^{2}} \sum_{i<k} \log \left(W_{i}-W_{k}\right) \log \left(\bar{W}_{i}-\bar{W}_{k}\right)
$$

To obtain the full expression compatible with $\mathcal{N}=4$ supersymmetry, we need to generalize this expression to include hypermultiplet couplings. This is needed to study backgrounds corresponding to D-branes moving in arbitrary directions. In order to do this, we first expand in $\mathcal{N}=1$ superfields. It may be seen by examining the structure of the one-loop diagrams that the correct general expression is obtained by rotating each of the terms in the sum (2.6) independently with respect to the manifest $S U(3) \times U(1)$ subgroup of the $S U(4)_{R}$ symmetry group of $\mathcal{N}=4$ supersymmetry. As expected from the supergravity point of view, the result corresponds to the pairwise interaction of D-threebranes.

It should be noted the one-loop exactness of $\mathcal{H}$ extends immediately to arbitrary gauge groups. It is then a simple matter to carry over the calculation above to obtain $\mathcal{H}$ at a generic point in the moduli space.

There is a singularity in $\mathcal{H}$ (2.6) when $W_{i} \rightarrow W_{k}$, which corresponds to nonabelian gauge symmetry being restored. In this limit, off-diagonal degrees of freedom become massless and should be included in the effective action. The $\mathcal{H}^{0}$ term in (1.2) will in general be non-zero in this situation.

After this work was completed, we received [14, 15] where similar results are obtained for the one-loop contribution to $\mathcal{H}(W, \bar{W})$.

\section{Acknowledgments}

D.L. thanks the Aspen Center for Physics for hospitality while this work was initiated. The research of D.L. is supported in part by DOE grant DE-FE0291ER40688-Task A.

\section{Note Added:}

The claim of this paper that we have determined the full perturbative and nonperturbative form of the dimension four operator $\mathcal{H}$ is not correct. First let us review the argument why the non-renormalization argument works for $S U(2)$ gauge group. 
Under both $U(1)_{R}$ and scale transformations terms in $\mathcal{H}$ of the form

$$
f(\tau, \bar{\tau}) \ln (W) \ln (\bar{W})
$$

are not invariant but get shifted. The shifted pieces are purely holomorphic or antiholomorphic and so are killed by the $N=2$ superspace measure. However, when $\tau$ is promoted to a background superfield this procedure does not work since the measure no longer kills the shifted piece. This is why such a term has to be independent of $\tau$ and thus is not renormalized.

For groups of higher rank however, one can construct several differnt manifestly invariant combinations of fields. Namely, the variables $\frac{W_{i}-W_{k}}{W_{j}-W_{l}}$ are all both $U(1)_{R}$ and scale invariant. This means that any function of these variables multiplied by a function of $\tau$ will be both scale and $U(1)_{R}$ invariant and hence we cannot exclude such contributions. Even when $\tau$ is promoted to a background superfield, such terms can be generated since they are manifestly invariant.

What we have shown is that the one-loop contribution is given by the $\mathcal{H}$ in the text and that terms of that particular form are not renormalized neither perturbatively nor non-perturbatively. 


\section{References}

[1] B. de Wit, M.T. Grisaru and M. Rocek, "Nonholomorphic corrections to the one-loop $\mathcal{N}=2$ super Yang-Mills action," Phys. Lett. B374 (1996) 297, hep-th/9601115.

[2] M. Dine and N. Seiberg, "Comments on Higher Derivative Operators in Some SUSY Field Theories," Phys. Lett. B409 (1997) 239, hep-th/9705057.

[3] N. Dorey, V.V. Khoze, M.P. Mattis, J. Slater and W.A. Weir, "Instantons, HigherDerivative Terms, and Nonrenormalization Theorems in Supersymmetric Gauge Theories", Phys. Lett. B408 (1997) 213, hep-th/9706007.

[4] D. Bellisai, F. Fucito, M. Matone and G. Travaglini, "Non-holomorphic terms in N=2 SUSY Wilsonian actions and RG equation" Phys. Rev. D56 (1997) 5218, hepth/9706099.

[5] V. Periwal and R. von Unge, Phys. Lett. B430 (1998) 71, hep-th/9801121.

[6] F. Gonzalez-Rey and M. Rocek, "Nonholomorphic $\mathcal{N}=2$ terms in $\mathcal{N}=4$ SYM: 1-loop calculation in $\mathcal{N}=2$ superspace," Phys. Lett. B434 (1998) 303, hep-th/9804010.

[7] I.L. Buchbinder and S.M. Kuzenko, "Comments on the background field method in harmonic superspace: non-holomorphic corrections in $N=4$ SYM," Mod. Phys. Lett. A13 (1998) 1623, hep-th/9804168.

[8] W. Taylor, "Lectures on D-branes, Gauge Theory and M(atrices)," hep-th/9801182.

[9] J. Maldacena, "The Large N Limit of Superconformal Field Theories and Supergravity," ATMP 2 (1998) 231, hep-th/9711200.

[10] S. Paban, S. Sethi and M. Stern, "Summing Up Instantons in Three-Dimensional Yang-Mills Theories," hep-th/9808119.

[11] S. Paban, S. Sethi and M. Stern, "Constraints From Extended Supersymmetry in Quantum Mechanics," hep-th/9805018.

[12] D.A. Lowe, "Constraints on Higher Derivative Operators in the Matrix Theory Effective Lagrangian," hep-th/9810075.

[13] N. Seiberg, "Naturalness versus supersymmetric non-renormalization theorems," hepph/9309335, Phys. Lett. B318 (1993) 469.

[14] F. Gonzalez-Rey, B. Kulik, I.Y. Park and M. Rocek, "Self-Dual Effective Action of $N=4$ Super-Yang Mills," hep-th/9810152, v2.

[15] E.I Buchbinder, I.L. Buchbinder and S.M. Kuzenko, "Non-holomorphic effective potential in $N=4 S U(n)$ SYM," hep-th/9810239. 